\title{
Selbstorganisierende Generierung strukturierter Prozeßmodelle
}

\author{
Peter Marenbach, Kurt Dirk Bettenhausen, Darmstadt, \\ und Bernd Cuno, Frankfurt/M.
}

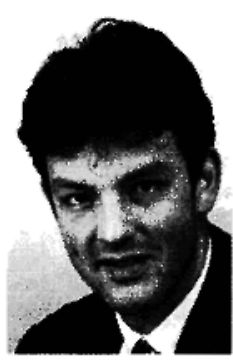

Dipl.-Ing. Peter Marenbach ist wissenschaftlicher Mitarbeiter am Institut für Regelungstechnik, Fachgebiet Regelsystemtheorie \& Robotik (Leiter: Prof. Dr. rer nat. Dipl.-Ing. Henning Tolle), der Technischen Hochschule Darmstadt. Hauptarbeitsgebiet: Anwendung von wissensbasierten und lernenden Systeme zur Autonomieerhöhung technischer Systeme, insbesondere zur Führung biotechnologischer Prozesse.

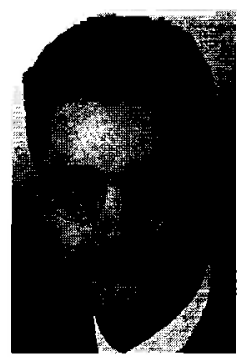

Dipl.-Ing. Kurt Dirk Bettenhausen ist wissenschaftlicher Mitarbeiter am Institut für Regelungstechnik, Fachgebiet Regelsystemtheorie \& Robotik (Leiter: Prof. Dr rer. nat. Dipl-Ing. Henning Tolle), der Technischen Hochschule Darmstadt. Hauptarbeitsgebiet: Integration von konventionellen sowie wissensbasierten und lernenden Methoden der Künstlichen Intelligenz (KI) zur Autonomieerhöhung technischer Systeme, insbesondere bei der Führung biotechnologischer Prozesse. Adresse: Technische Hochschule Darmstadt, Institut für Regelungstechnik, Fachgebiet Regelsystemtheorie \& Robotik, Landgraf-Georg-Stráße 4, D-64283 Därmstadt, Tel.: (06151) 163442

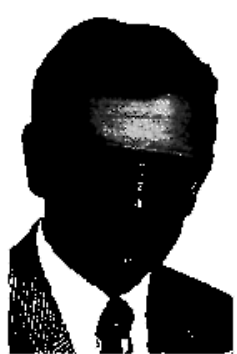

Prof. Dr.-Ing. Bernd Cuno ist Leiter der Abteilung Technische Planung und Koordination im Geschäftsleld Automatisierungstechnik der AEG Aktiengesellschaft in Frankfurt am Main und Honorarprofessor an der Fachhochschule Darmstadt. Hauptarbeitsgebiete: Ermittlung zukünftiger Marktanforderungen, Bewertung neuer Technologien sowie Transfer von Forschungsergebnissen in innovative Produkte der industriellen Automatisierungstechnik.

Dèr Beiträg stellt einen neuartigen Ansātz zur automatischen Erzeugung beliebiger strukturierter Prozeßmodelle vor, der es erlaubt, den Ingenieur bei einem der zeitaufwendigsten im Rahmen der Automatisierung komplexer Prozesse anfallenden Arbeiten - der Modellierung dess Prozesses - effizient zu unterstützen.

Ausgehend von den grundlegenden Ideen des ,,genetischen Programmierens" wurde ein Konzept für ein wirkungsvolles Werkzeug für den Ingenieur entwickelt, das am Beispielproze $\beta$ einer Fluß-Stauhaltung experimentell untersucht wurde.

Self-organizing generation of structured process models

The article at hand presents a new approach for the automatic development of any structured process model which supports the engineer in one of the most time-expensive tasks during automation of complex processes - the process modelling.
Based on the fundamental ideas of "genetic programming" a conception for an effective tool was developed and some experimental studies concerning the sample process of a river barrage system were performed.

\section{Einleitung}

Ein Prozeßmodell läßt sich formal als eine Formulierung des Wissens über das Verhalten und/oder die Struktur des betrachteten Prozesses definieren. Die Form, die für die Darstellung dieses Wissen angewendet wird, ist dabei in großem Maße von der gegebenen Aufgabenstellung abhängig.

In der Regelungs- und Automatisierungstechnik werden Prozeßmodelle vor allem als Ausgangspunkt für den Entwurf und die Auslegung von Reglern benötigt. Der größte Teil der gebräuchlichen Darstellungsformen $-\mathbf{z}$. B. die Übertragungsfunktion im Frequenzbereich oder die Zustandsraumdarstellung - beruht auf linearisierten Differentialgleichungssystemen und wird in Form eines strukturierten mathematischen Modells formuliert. Zur Synthese von Reglern für die unterschiedlichsten Aufgaben und Anforderungen auf der Basis solcher linearer Modelle existiert eine umfassende Theorie (siehe z.B. [1]).

Eine ganz andere Klasse von ProzeBmodellen sind die unstrukturierten, direkten Eingangs-/Ausgangsmodelle. Diese Modelle geben die im allgemeinen nichtlineare Abbildung der Eingangsgrößen auf die Ausgangsgrößen lediglich in Form einer ,,Black Box ${ }^{i *}$ wieder und bieten keine übersichtlichen Informationen über die inneren Zustände und das grundsätzliche Verhalten des betrachteten Prozesses. Aus diesem Grund lassen sich anhand solcher Modelle nur schwer Aussagen, z. B. über den günstigen Aufbau einer Regelung, machen. Andererseits können prädiktive Eingangs-/ Ausgangsmodelle in lernenden Regelkreisen zum Generieren von Regelstrategien verwendet werden (siehe z. B. [2]).

Die Methoden der Modellbildung sind bei den beiden hier vorgestellten Modelltypen grundlegend verschieden. Bei strukturierten mathematischen Modellen geht man in der Regel analytisch vor, d.h., man wählt entweder einen deduktiven Weg, ausgehend von allgemeinen, den Prozeß beschreibenden naturwissenschaftlichen Gesetzmäßigkeiten, oder den induktiven 
- auch als datengetrieben bezeichneten - Weg, ausgehend von dem beobachteten bzw. gemessenen Prozeßverhalten. Der Einsatz von Identifikationsverfahren (siehe z. B. [3]) ist ein effizienter Ansatz zur Anpassung strukturierter mathematischer Modelle an einen Prozeß durch die Schätzung der Prozeßparameter anhand gemessener Prozeßdaten, der es jedoch nicht gestattet, bekannte Teilmodelle selbständig zu ergänzen, vorgegebene Strukturen zu verändern oder gar neue Strukturen zu erzeugen.

Bei Kennfeldspeichern, Neuronalen Netzen oder Assoziativspeichern, die geeignet sind, das Prozeßverhalten in Form eines Eingangs-/Ausgangsmodells nachzubilden, erfolgt die Modellbildung auf einem ganz anderen Weg. Anhand von Prozeßdaten wird das Modellverhalten entweder direkt abgespeichert oder schrittweise erlernt. Die Qualität solcher erlernter Modelle hängt in großem Maße von den Trainingsdaten ab. Da direkte Eingangs-/Ausgangsmodelle nicht die Transparenz strukturierter mathematischer Ausdrücke bietet, läßt sich an einem trainierten Modell nicht erkennen, ob es das gewünschte Prozeßverhalten allgemein wiedergibt, oder ob es sich nur um eine auf den speziellen Datensatz zugeschnittene Lösung handelt. Ein weiterer Nachteil von solchen einfachen Eingangs-/Ausgangsmodellen ist ihr im allgemeinen geringer Prädiktionshorizont, d.h., daß man mit Hilfe eines solchen Modells nur bedingt Aussagen über das in entfernter $\mathrm{Zu}$ kunft zu erwartende Verhalten machen kann. Derartige Aussagen sind bei der Führung von nichtkontinuierlichen Prozessen, z. B. Batch-Fermentationen in der Biotechnologie, von besonderem Interesse, da vielfach schon das Anfangsstadium des Prozesses entscheidend für den gesamten späteren Verlauf ist.

In diesem Beitrag wird ein Ansatz vorgestellt, der es ermöglicht, selbstorganisierend strukturierte mathematische Modelle zu generieren, und damit die Fähigkeit zum Selbstlernen, wie sie von direkten Eingangs-/ Ausgangsmodellen her bekannt ist, mit der Transparenz und der guten Handhabbarkeit, die man an den klassischen Darstellungsformen für Modelle schätzt, zu vereinen. Dabei müssen weder Struktur noch Parameter a priori bekannt sein. Ist allerdings Vorwissen über das zu erwartende Modell vorhanden, so erlaubt es der hier vorgestellte Ansatz, dieses Wissen in den Selbstorganisationsproze $B$ einfließen zu lassen. Die Darstellung der Modelle erfolgt in Form von Blockschaltbildern, die aus elementaren, in der Regelungstechnik gebräuchlichen linearen Übertragungsblökken, wie Verzögerungs-, Verstärkungs- und Totzeitgliedern, sowie nichtlinearen Elementen, wie Schaltern und Multiplikationsstellen, zusammengesetzt werden.

Im Abschnitt 2 wird zunächst die grundlegende Idee des Ansatzes und im Abschnitt 3 wesentliche Aspekte der Realisierung vorgestellt, bevor im Abschnitt 4 der betrachtete Beispielprozeß einer Fluß-Stauhaltung und im Abschnitt 5 die daran durchgeführten Untersuchungen beschrieben werden. Der Beitrag schließt mit einer Zusammenfassung und einem kurzen Ausblick auf weitere, im Rahmen dieses Projektes vorgesehene Tätigkeiten.

\section{Ansatz}

Bild 1 zeigt den prinzipiellen Ansatz, der es ermöglicht, in gleicher Weise sowohl prozeßbezogenes Bedienerwissen als auch konkrete Prozeßdaten zur Modellbildung heranzuziehen. Der Bediener hat zum einen die Möglichkeit, im Sinne der Constraint Propagation [4] bekannte Teilmodelle oder feststehende Zusammenhänge zu erzwingen, und zum anderen kann er durch Vorgabe des Inhalts eines „Modellbaukastens“" bestimmen, welche Übertragungsblöcke zur Generierung des Modells verwendet werden sollen und welche nicht. Die Prozeßdaten werden zum einen dazu herangezogen, die in einem strukturierten Modell enthaltenen Parameter und Koeffizienten einzustellen. Zum anderen dienen sie auch zur Bewertung einer vorliegenden Modellstruktur. Diese Bewertung erfolgt, indem das mit einem Trainingssignal beaufschlagte Modell simuliert und das sich dabei ergebende Verhalten mit dem bekannten Soll-Prozeßverhalten verglichen wird. Die Bewertung des vorliegenden Modells dient als lenkende Kraft für eine iterative Verbesserung durch schrittweise Veränderung und Ergänzung der Struktur.

Bild 1: Ansatz zur selbstorganisierenden Modellbildung.

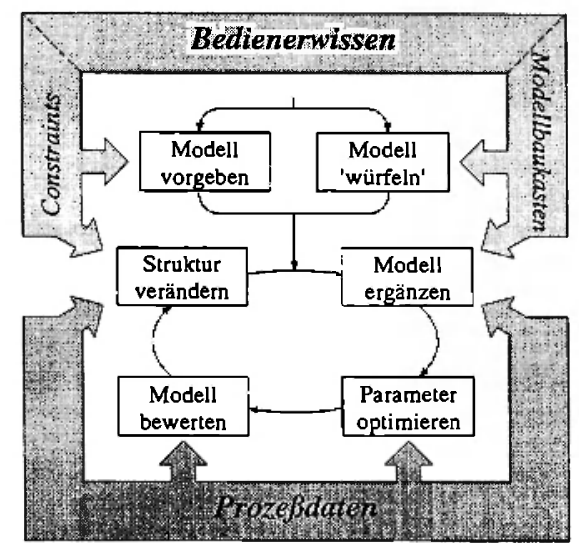

Die oben beschriebene Aufgabenstellung, in der ausgehend von Prozeßdaten ein Blockschaltbild konstruiert werden soll, das dem angestrebten Kompromi $\beta$ zwischen der exakten Wiedergabe des Prozeßverhaltens und einer transparenten, gut handhabbaren Darstellung entspricht, stellt ein komplexes Optimierungsproblem dar.

Konventionelle, iterativ vorgehende Optimierungsverfahren arbeiten nach dem Prinzip, daß durch Gradientenbildung oder durch Ausprobieren die Richtung im Suchraum ermittelt wird, in der sich die größten Verbesserungen erzielen lassen. Die zu optimierenden Größen werden dann schrittweise in die gefundene Richtung verändert. Bei der Suche nach optimalen Parametern kann - auch bei hochdimensionalen Problemen - auf eine große Zahl bekannter und bewährter Verfahrèn zurückgègriffen werden (siehe z. B. [5]). Im Zusammenhang mit Strukturen, die im Rahmen der vorliegenden Aufgabenstellung optimiert werden sollen, sind diese Verfahren allerdings nicht anwendbar, da sich für Strukturen keine Ordnungsrelationen und somit keine Richtungen im Suchraum definieren lassen. 
Optimierungsverfahren, die auf der Nachahmung des natürlichen Prinzips der Evolution aufbauen, basieren auf einem grundlegend anderen Ansatz. Solchen Verfahren, deren Vorgehensweise sich grob als zielgerichtetes Ausprobieren beschreiben läßt, erweisen sich zwar im direkten Vergleich mit den zuerst beschriebenen Verfahren als rechenaufwendig und damit zeitintensiv, doch lassen sie sich auch auf Probleme in einem nicht geordneten Suchraum anwenden.

\subsection{Evolutionäre Verfahren}

Schon 1859 formulierte Charles Darwin die Hypothese eines natürlichen Selektionsprozesses, nach der ein besser an seine Umwelt angepaßtes Lebewesen die höhere Überlebens- und Fortpflanzungswahrscheinlichkeit als ein nicht oder nur schlecht angepaßtes hat. Durch die daraus resultierende Selektion wird ein sich in kleinen Veränderungen äußernder Entwicklungsprozeß ohne explizite Kenntnis jeglicher innerer Struktur in Richtung von immer besser der Umwelt angepaßten Lebenwesen gesteuert. Einer der ersten bekannten Ansätze, diese Vorstellung von der natürlichen Evolution für technische Optimierungsprobleme nutzbar zu machen, ist die von Rechenberg [6] veröffentlichte Evolutionsstrategie. Unabhängig von Rechenberg entwikkelte Holland [7] einen ähnlichen Ansatz, dessen Bezeichnung Genetische Algorithmen sich heute international für alle Arbeiten auf dem Gebiet der evolutionären Optimierung durchgesetzt hat.

Die prinzipielle Vorgehensweise bei evolutionären Verfahren läßt sich durch die folgenden fünf Schritte beschreiben:

1. Erstellen einer Anfangspopulation von verschiedenen Lösungen; dies erfolgt in der Regel zufällig.

2. Bewertung aller Individuen der Population. Als Maß dafür, inwieweit eine Lösung den gestellten Anforderungen entspricht, wird ein Fitne $\beta$-Wert bestimmt.

3. Selektion der Eltern für die nächste Generation. In diesem Schritt kommt das Darwin'sche Prinzip zur Anwendung; Individuen mit einer hohen Fitneß werden mit großer Wahrscheinlichkeit gewählt.

4. Erstellen einer Population von Nachkommen. Aus den im vorangegangenen Schritt ausgewählten Individuen werden mit Hilfe sogenannter genetischer Operatoren die Individuen einer neuen Generation erzeugt.

5. Mit der neuen Population zurück zu 2.

Sowohl die Evolutionsstrategie als auch die Genetischen Algorithmen wurden ursprünglich genau wie die oben beschriebenen konventionellen Methoden zur Optimierung von Parametern entworfen [8]. Kennzeichnend hierfür ist, daß jedes der betrachteten Individuen durch einen Ausdruck konstanter Länge repräsentiert wird.

Eine andere Art der Repräsentation wählte Koza [9] für einen Ansatz, den er als genetisches Programmieren bezeichnet. Anstelle der Betrachtung eines reinen Parametervektors, besteht ein Individuum bei Kozas Ansatz aus einer aus Operatoren und Variablen zusammengesetzten Baumstruktur, die sich als eine Art Computer- programm interpretieren läßt (daher die Bezeichnung ,genetisches Programmieren“). Durch diese Darstellungsart lassen sich strukturierte Ausdrücke variabler Länge erstellen und optimieren.

Koza zeigt am Beispiel einer Fourier-Reihenzerlegung, wie man mit Hilfe dieses Verfahrens eine strukturierte mathematische Darstellung eines vorgegebenen Kurvenverlaufs erzeugen kann. Bei diesem Beispiel zeigt sich allerdings ebenso die große Schwäche dieses Ansatzes. $\mathrm{Zu}$ einer Fourier-Reihenzerlegung gehört nicht nur die Auswahl der zu berücksichtigenden Oberwellen, d.h. die Wahl der richtigen Struktur, sondern auch das Bestimmen der Koeffizienten jeder Teilschwingung. Die von Koza in diesem und in anderen Beispielen vorgeschlagene Vorgehensweise zur Erzeugung solcher Koeffizienten ist, daß man eine bestimmte Anzahl zu Beginn zufällig bestimmter Konstanten als Operatoren definiert. Aus diesen Konstanten in Verbindung mit arithmetischen Operatoren soll der Algorithmus die richtigen Koeffizienten zusammensetzen. Dies führt schon bei einfachsten Aufgabenstellungen zu extrem langen Ausdrücken, die erst nach umfangreichen Umformungen und dem Zusammenfassen der Konstanten den Blick auf die erstellte Struktur freigeben.

\subsection{Vorgehensweise}

Ein strukturiertes Prozeßmodell besteht aus einer die Zusammenhänge wiedergebenden Struktur sowie den in dieser Struktur enthaltenen Parametern und Koeffizienten. Betrachtet man die ingenieurmäßige Vorgehensweise beim induktiven Erstellen eines solchen Modells, so erfolgt erst die Wahl einer möglichen Struktur und danach die Einstellung der Parameter. Die im vorangegangenen Abschnitt vorgestellten Optimierungsverfahren, die entweder zur Adaption von Parametern und nicht von Strukturen oder nur zum Auffinden günstiger Strukturen aber nicht zum Optimieren von Parametern geeignet sind, legen es nahe, auch für die selbstorganisierende Modellbildung eine derartige zweistufige Strategie anzuwenden. Im Bild 2 ist die sich so ergebende Vorgehensweise bei der selbstorganisierenden Generierung strukturierter Prozeßmodelle in Form

Bild 2: Vorgehensweise.

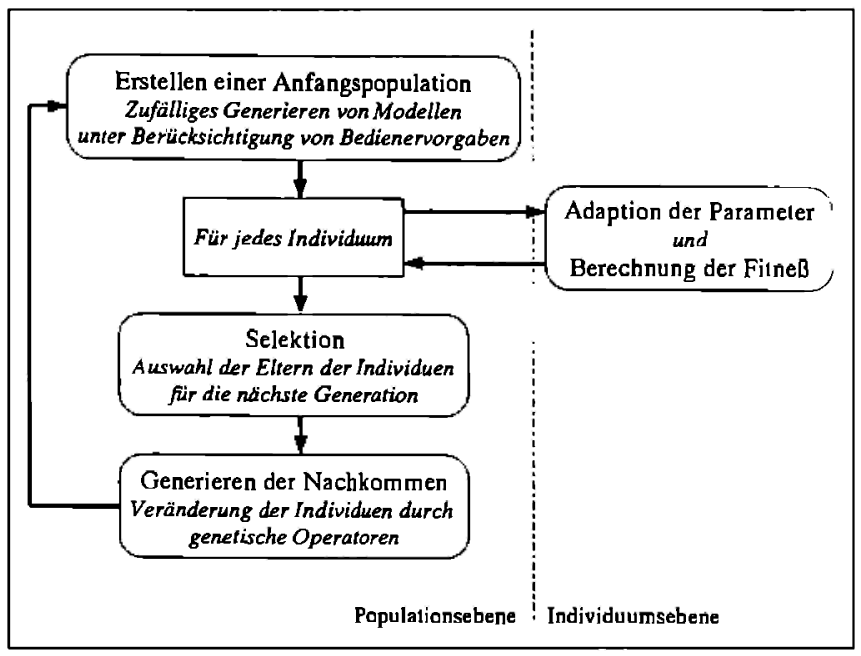


eines Ablaufdiagramms dargestellt. Man erkennt links den evolutionären Algorithmus zur Struktursuche, die auf der Populationsebene stattfindet, und rechts die $\mathrm{Pa}$ rameteradaption, für die bislang ein konventionelles Optimierungsverfahren auf der Ebene des einzelnen Individuums eingesetzt wird.

\section{Realisierung}

In diesem Abschnitt sollen nun die einzelnen im Bild 2 angeführten Schritte näher erläutert werden. $\mathrm{Zu}$ nächst wird allerdings die Repräsentationsform vorgestellt, die zur Darstellung der Blockschaltbilder gewährt wurde, um sie in der Art des ,,genetischen Programmierens" verarbeiten und optimieren zu können.

\subsection{Repräsentation}

Im Abschnitt 2.1 wurde erwähnt, daß bei der ,genetischen Programmierung" nach Koza Operatoren und Variablen zu strukturierten baumförmigen Ausdrücken zusammengefügt werden. Für die Aufgabenstellung der Modellbildung werden nun eine Auswahl elementarer Übertragungsglieder als Operatoren und die Eingangsgrößen des zu modellierenden Prozesses als gleichartige Variablen definiert.

Jeder Modellblock (Übertragungsglieder oder Eingangsgröße) entspricht einem Gen. Die genetische Darstellung eines Modell-Individuums erfolgt durch eine Kette von solchen Genen, die sich in Anlehnung an die Biologie auch als Chromosom bezeichnen läßt. Das einzelne Gen enthält neben der Information darüber, welches Übertragungsglied es repräsentiert, die Anzahl der Eingänge des entsprechenden Modellblocks und die numerischen Werte der in dem Modellblock enthaltenen Parameter. Im Bild 3 ist ein Beispiel für einen genetischen Ausdruck, der aus fünf solchen Genen zusammengesetzt ist, dargestellt. In der ersten Zeile jedes Gens ist ein symbolischer Bezeichner für das Übertragungsglied, das durch das jeweilige Gen repräsentiert wird, angegeben. Hierbei steht ADD für ein Additionsglied, PT 2 für ein Verzögerungsglied zweiter Ordnung, $T t$ für ein Totzeitglied, und $\mathrm{u} 1$ sowie $\mathrm{u} 2$ repräsentieren je eine Eingangsgröße. In der zweiten Zeile ist jeweils ein Zeichen angegeben, das bei der internen Verarbeitung verwendet wird, und die Anzahl der Eingänge in den Modellblock stehen jeweils in der dritten Zeile. Bei dem Verzögerungs- sowie bei dem Totzeitglied sind außerdem im unteren Teil die zugehörigen Parameter mit den in der Regelungstechnik üblichen Bezeichnungen angegeben.

Bild 3: Genetischer Ausdruck bestehend aus fünf Genen.

\begin{tabular}{|l|l|l|l|l|}
\hline "ADD" & "u1" & "PT2" & "Tt" & "u2" \\
'A' & '1' & 'F' & 'J' & '2' \\
2 & 0 & 1 & 1 & 0 \\
\hline- & - & $\mathrm{K}=$ & $\mathrm{Tt}=$ & - \\
& & $\begin{array}{l}\mathrm{N}= \\
\mathrm{d}=\end{array}$ & & \\
\hline
\end{tabular}

Bild 4: Darstellung als Baumstruktur.

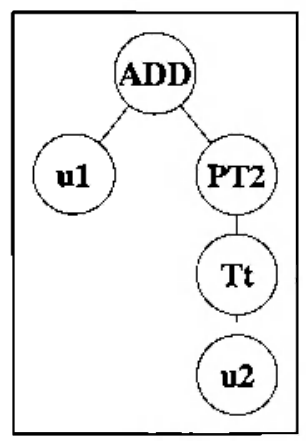

Aufgrund der in jedem Gen angegebenen Anzahl von Eingängen läßt sich der im Bild 3 dargestellte Ausdruck in die im Bild 4 gezeigte Baumstruktur überführen. Diese Baumstruktur wird vor allem zur rechnerinternen Behandlung des Modells benötigt. Im Bild 5 ist schließlich die äquivalente Darstellung in Form des für den Regelungstechniker interessanten Blockschaltbildes dargestellt.

u1

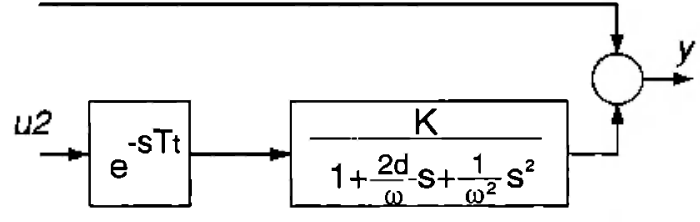

Bild 5: Darstellung als Blockschaltbild.

\subsection{Erstellen einer Anfangspopulation}

Als Ausgangspunkt der Modellsuche wird zunächst eine möglichst variantenreiche Anfangspopulation benötigt. Im einfachsten Fall werden die Individuen für die Anfangspopulation rein zufällig zusammengesetzt. Dabei werden nach und nach Modellblöcke aus dem „Modellbaukasten" entnommen, bis sich ein geschlossener Ausdruck ergibt. Für die Parameter, die zu den einzelnen Modellblöcken gehören, werden ebenfalls zufällig Anfangswerte bestimmt.

Ergänzend zu dem rein zufälligen Generieren der Individuen, gibt es eine Reihe von optionalen Möglichkeiten, Vorwissen über den Prozeß oder über die erwartete Struktur in die Anfangspopulation einzubringen. Der Bediener kann

- den Inhalt des „Modellbaukastens" vorgeben,

- die Intervalle, aus denen die Anfangsparameter zufällig gewählt werden, vorgeben bzw. variieren,

- feste Blocksequenzen, die intern wie ein einzelner Modellblock behandelt werden, definieren (Constraint Encapsulation) und/oder

- für alle Individuen verbindliche Anfangssequenzen im Sinne von Teilmodellen vorgeben.

\subsection{Adaption der Parameter}

Um eine gute Übereinstimmung zwischen dem gespeicherten Prozeßverhalten und der Antwort, die sich aus der Simulation des Modells ergibt, zu erreichen, müssen die zunächst zufällig gewählten, in den strukturierten Modellen auftretenden Parameter, an den 
Prozeß adaptiert werden. Bislang wird dazu das Verfahren nach Hooke und Jeeves [10], ein iterativ arbeitendes Parametersuchverfahren, verwendet. Dieses Verfahren zeichnet sich dadurch aus, da $B$ es von den Anfangswerten ausgehend relativ zügig und robust in das nächstgelegene lokale Optimum läuft. Für jede Iteration der Parametersuche werden die folgenden vier Schritte durchlaufen:

1. Simulation des Modells anhand des Trainingsdatensatzes,

2. Ermittlung der mittleren quadratischen Abweichung zum vorgegebenen Sollverhalten,

3. Vergleich des aktuellen mit früher mit anderen $\mathrm{Pa}$ rametern erreichten Ergebnissen,

4. Variation der Parameter in die Richtung, in der ein Erfolg erzielt wurde, bzw. Suche nach einer neuen günstigen Richtung.

Aufgrund der bei jedem Iterationsschritt nötigen Simulation (Schritt 1) ist die Adaption der Parameter sehr zeitintensiv und bestimmt wesentlich die Gesamtlaufzeit des Verfahrens. Da hierbei aber immer nur ein einzelnes Individuum unabhängig von der restlichen Population betrachtet wird - man spricht hier auch von einer inheränten Parallelität [8] -, bietet es sich an, sofern entsprechende Möglichkeiten vorhanden sind, diese Berechnungen auf parallele Hardware abzubilden.

Bild 6 zeigt eine beispielhafte, mit der vorliegenden Implementierung des Verfahrens erreichbare Konfiguration aus elf auch räumlich verteilten SUN-SparcStations des Fachgebiets Regelsystemtheorie \& Robotik an der TH Darmstadt. Man erkennt, daß auf einem zentralen Rechner das Programm gen, in dem die evolutionäre Struktursuche und die Koordination des Gesamtsystems stattfinden, und auf allen anderen jeweils das Programm calc (im Bildschirm der symbolisch dargestellten Rechner angedeutet), das zur Optimierung der Parameter je eines Individuums dient, läuft. Der Ablauf der Bestimmung der Fitneß einer Generation erfolgt in diesem Rechnerverbund derart, daß zunächst alle Programme calc mit der Berechnung eines Indivi- duums beauftragt werden und $\mathrm{da} \beta$ im folgenden, jeweils sobald ein Fitneßwert geliefert wird, auf dem frei gewordenen Rechner die Berechnung eines weiteren Individuums gestartet wird.

\subsection{Berechnung der Fitneß}

Die Fitneß eines Individuums ist einer der zentralen Begriffe der evolutionär motivierten Suchverfahren. Koza [9] bezeichnet die Fitneß als die treibende Kraft der Darwin'schen Hypothese und in gleicher Weise der genetischen Algorithmen und des, genetischen Programmierens". Die FitneB macht eine Aussage darüber, wie gut ein Individuum den gestellten Anforderungen genügt, und ist die von dem evolutionären Verfahren zu maximierende Größe. Im wesentlichen gleicht die Vorschrift für das Ermitteln der Fitneß einem Gütekriterium, wie es von anderen Optimierungsverfahren her bekannt ist.

Wie eingangs schon bemerkt, ist es bei dem Erstellen eines strukturierten mathematischen Prozeßmodells nötig, einen Kompromiß zwischen einer möglichst exakten Wiedergabe des Prozeßverhaltens einerseits und einer übersichtlichen und handhabbaren Struktur andererseits zu finden. Entsprechend müssen diese beiden Faktoren mathematisch formuliert in die Berechnung der Fitneß eingehen. Dazu wurde ein Fehlermaß $E_{i}$, das die mittlere quadratische Abweichung zwischen Ist- und Sollverlauf beschreibt, und ein Komplexitätsmaß $K_{i}$, das eine Aussage über Anzahl und Art der verwendeten Modellbausteine macht, definiert. Die Fitne $B f_{i}$ eines Individuums $i$ wird aus diesen beiden Maßzahlen nach

$$
f_{i}=\frac{1}{1+E_{i} K_{i}}
$$

berechnet. (1) ist eine heuristische Formel, die die Einhaltung des Kompromisses zwischen Fehlermaß und Komplexität sicherstellen soll. Im Rahmen der Untersuchungen wurden auch andere Berechnungen der Fitneß eingeführt, bislang konnten mit diesen aber keine besseren Ergebnisse erzielt werden.
Bild 6: Verteiltes Rechnen am Fachgebiet Regelsystemtheorie \& Robotik der TH Darmstadt

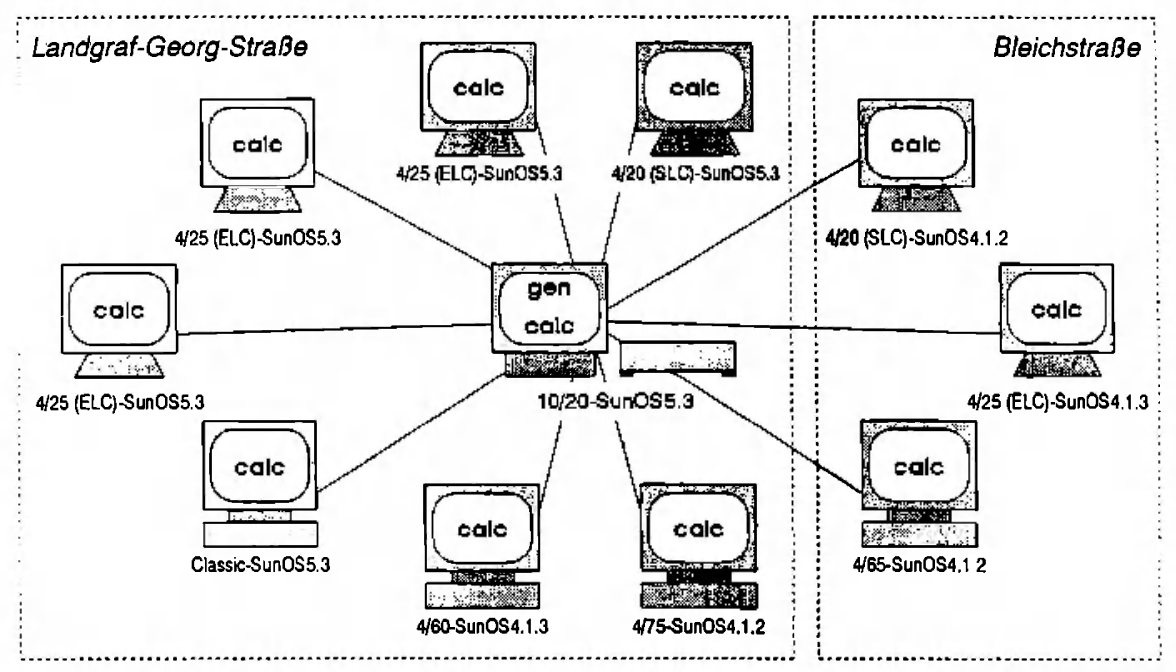

\subsection{Selektion}

Die grundlegende Idee bei evolutionären Optimierungs̄methoden ist, da $B$, in Anlehnung an Darwins Hypothese, die Individuen mit hoher Fitneß eine entsprechend hohe Fortpflanzungswahrscheinlichkeit haben, die sich wiederum in einer hohen Anzahl von Nachkommen äußert. Der in dem evolutionären Verfahren verwendete Selektionsmechanismus hat die Aufgabe, auf Basis dieses Prinzip jeweils als Ausgangspunkt eines Nachkommens ein Eltern-Individuum aus der aktuellen Population auszuwählen. Im Zusammenhang mit Genetischen Algorithmen finden sich in 
der Literatur die unterschiedlichsten Mechanismen, mit denen diese Selektion erfolgen kann. Da der Selektionsmechanismus völlig unabhängig von der Art der Repräsentation der Individuen allein anhand der Fitneß arbeitet, können diese Mechanismen in der gleichen Form beim ,genetischen Programmieren" und bei dem hier verfolgten Ansatz angewendet werden. Das Standardverfahren, das von Holland für die Genetischen Algorithmen vorgeschlagen wurde, wird als fitneß-proportionale Selektion bezeichnet. Wie der Name schon ausdrücken soll, ist hierbei die Wahrscheinlichkeit, da $\beta$ ein bestimmtes Individuum ausgewählt wird, direkt proportional zu seiner Fitneß. Im Englischen wird dieses Verfahren mitunter auch als roulette-wheel-selection bezeichnet, da man sich vorstellen kann, daß die Selektion mittels eines Glücksrades erfolgt, auf dem jedes Individuum einen Sektor zugeteilt bekommt, dessen Größe von seiner Fitneß abhängt.

Goldberg [11] hat die gebräuchlichsten Selektionsmechanismen gegenübergestellt und bezüglich ihres Einflusses auf die „Konvergenz" der evolutionären Optimierung und die beanspruchte Rechenzeit untersucht. Dabei zeigte sich, daß die fitneßproportionale Selektion, wenngleich sie im Blick auf das Vorbild der natürlichen Evolution recht plausible erscheint, wesentliche Schwächen hat. Entsteht in frühen Generationen, in denen die durchschnittliche Fitneß der Population noch gering ist, ein herausragendes Individuum, dessen Fitne $B$ weit über der der anderen Individuen liegt, so treten es und seine Nachkommen so massiv in den folgenden Generationen auf, daß die Vielfalt und damit die Kreativität des evolutionären Prozesses leidet. Liegen andererseits in späten Generationen die Fitneßwerte der Individuen auf hohen Niveau dicht beieinander, so ist auch die Reproduktionsrate aller Individuen nahezu gleich und das Verfahren geht in eine blinde Suche über.

Aus diesem Grund kommt bei dem hier vorgestellten Verfahren die Turnier-Selektion, die auf eine unveröffentlichte Arbeit von Wetzel zurück geht [11], zur Anwendung. Hierbei werden zunächst zwei oder auch mehr Individuen zufällig aus der Population ausgewählt. Dasjenige Individuum, das von dieser Gruppe die größte Fitneß hat, wird zur Reproduktion herangezogen. Auf diese Weise ist für die Reproduktionswahrscheinlichkeit eines Individuums allein die Position, die es in einer Fitneß-Rangliste der Population einnimmt, und nicht das Verhältnis, in dem seine Fitneß zu der der anderen Individuen steht, entscheidend. Mathematisch läßt sich die Wahrscheinlichkeit $P_{i}$ (Auswahl), daß ein Individuum $i$ in einem Selektionsschritt bei der Turnier-Selection ausgewählt wird, in Abhängigkeit von seiner Position ' $p_{i}$ innerhalb der gedachten Rangliste nach

$$
P_{i}(\text { Auswahl })=\sum_{j=1}^{k}\left(\frac{1}{n}\right)^{j}\left(\frac{n-p_{i}}{n}\right)^{k-j}\left(\begin{array}{l}
n_{j} \\
j
\end{array}\right)
$$

berechnen. Dabei bezeichnen $n$ die Populationsgröße und $k$ die Anzahl der Individuen, die zu einem ,,Turnier" herangezogen werden.

\subsection{Generieren der Nachkommen}

Die durch den Selektionsmechanismus bestimmten Eltern-Individuen werden, bevor sie in die Generation der Nachkommen übernommen werden, mit den sogenannten genetischen Operatoren modifiziert. Im Rahmen des hier vorgestellten Verfahrens kommen drei genetische Operatoren zur Anwendung, die auch von $\mathrm{Ko}$ za bei der ,genetischen Programmierung“ eingesetzt werden. Der erste und einfachste dieser Operatoren ist die Reproduktion, bei der das Eltern-Individuum direkt ohne Veränderung übernommen wird.

Ein weiterer genetischer Operator ist die Mutation, d.h. eine mehr oder weniger zufällige Veränderung des Individuums. Wie im Bild 7 dargestellt, wird bei der Mutation zunächst zufällig ein Modellblock des Individuums als Mutationspunkt bestimmt. Dieser wird dann durch einen zufälig aus dem Modellbaukasten gewählten anderen Modellblock ersetzt. Ist - wie in dem im Bild 7 dargestellten Beispiel - die Anzahl der Eingänge des alten Blocks ungleich der des neuen, so werden überzählige Äste entfernt bzw. fehlende Äste neu generiert (im Bild 7 der grau unterlegte Block $u 2$ ).

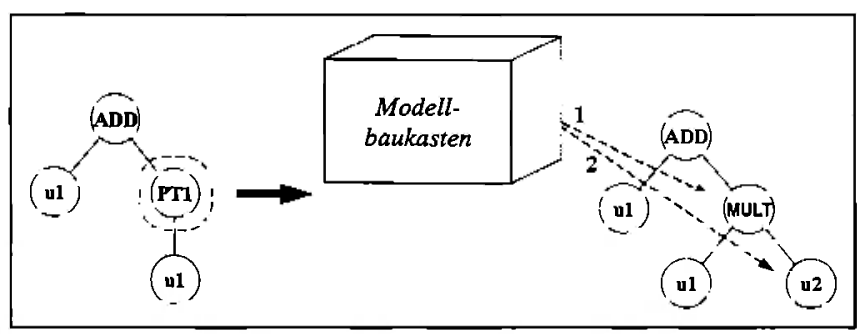

Bild 7: Funktionsweise der Mutation.

Der letzte und wichtigste genetische Operator bei der evolutionären Struktursuche ist die Rekombination auch crossover -, zu der im Gegensatz zu den beiden anderen Operatoren zwei Eltern herangezogen werden. Dies entspricht einer geschlechtlichen Fortpflanzung, wie sie auch bei allen hochentwickelten Organismen in der Natur angetroffen wird. Bild 8 zeigt die Funktionsweise des Rekombination: Zunächst wird in bei-

Bild 8: Funktionsweise der Rekombination.

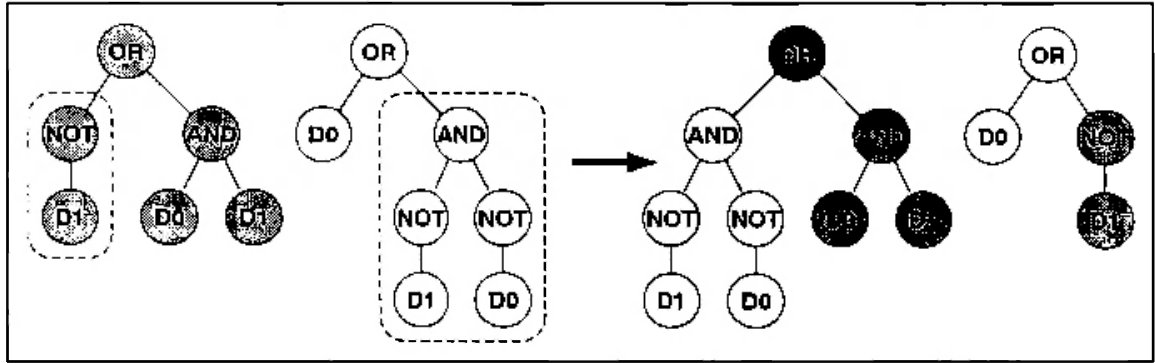


den Individuen wie bei der Mutation zufällig ein Modellblock bestimmt. Anschließend werden die von dem jeweiligen Block ausgehenden Teilausdrücke herausgetrennt und in das jeweils andere Individuum eingesetzt. Schließlich werden die beiden neu entstandenen Individuen in die Generation der Nachkommen übernommen.

\section{Beispielprozeß Flußstauhaltung}

Seit frühester Zeit nutzen Menschen die Flüsse nicht nur als Lieferanten für Wasser, sondern auch als Transportwege und Antriebsmittel. Schon die großen Flußkulturen am Nil, an Euphrat und Tigris sowie am Gelben Fluß besaßen zur Nutzung der Flüsse Einrichtungen, die zu den ältesten technischen Großanlagen der Menschheit gehören.

Obgleich die Nutzung der Flüsse als Verkehrsweg und zur Energieerzeugung vorübergehend an Bedeutung verloren hatte, so wird ihr heute, nicht zuletzt aus ökologischen Gesichtspunkten, wieder zunehmend Interesse gewidmet.

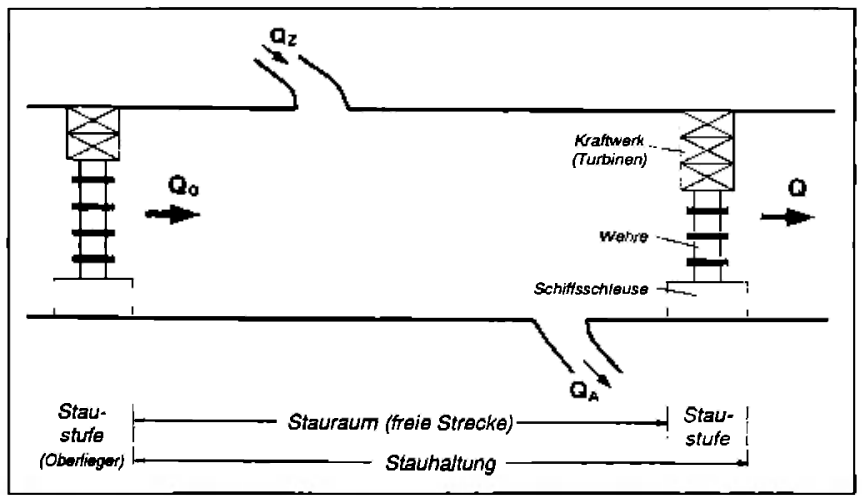

Bild 9: Grundri $B$ einer Stauhaltung ( $Q$ : AbfluB, $Q_{0}$ : Zufluß (Oberlieger-Abfluß), $Q_{Z}$ und $Q_{A}$ : stauhaltungsinterne Zu- bzw. Ábflüsse).

Bild 9 zeigt den schematischen Grundriß einer Flußstauhaltung. Als Stauhaltung bezeichnet man den gesamten Flußverlauf zwischen zwei Staustufen (die freie Strecke) einschließlich der Staustufe. Die Staustufe besteht aus den sogenannten Regelorganen sowie im allgemeinen einer Fischtreppe und bei schiffbaren Flüssen zusätzlich aus einer Schleusenanlage. Als Regelorgane werden die Wehre der Wehranlagen und, sofern vorhanden, die Turbinen des Flußkraftwerks bezeichnet. Die Stellgröße, die durch die Staustufe manipuliert werden kann, ist deren Abfluß $Q$, der sich aus der Summe der Abflüsse der Regelorgane ergibt. Die wesentlichen Störgrößen sind der Oberlieger-Abfluß $Q_{o}$ sowie stauhaltungsinterne $\mathrm{Zu}-$ flüsse $Q_{Z}$ und Abflüsse $Q_{A}$. Bild 10 zeigt einen Längsschnitt durch die Stauhaltung und die Benennung charakteristischer Elemente nach DIN 4048 und 4054.

\subsection{Motivation und Schwierigkeiten}

Die umfangreichen Anforderungen, die an moderne Flußstauhaltungen gestellt werden, sollen zunehmend durch vollautomatisierte Anlagen erfüllt werden. Ziele der dahingehenden Anstrengungen sind,

- die regenerative Energiequelle Wasserkraft möglichst effizient zu nutzen,

- die Verfügbarkeit der Flüsse und Kanäle als Transportwege stets sicherzustellen,

- im Rahmen der Rationalisierung sowie der Erleichterung der Betriebsführung einen vollautomatischen Betrieb mit Fernüberwachung zu gewährleisten und - die größtmögliche Betriebssicherheit zu garantieren.

Auf Basis dieser Motive für die Automatisierung von Stauhaltungen lassen sich die Aufgaben für die automatisierte Prozeßführung formulieren. Dies sind vor allem

- das Einhalten eines Oberwasserpegel-Sollwertes (vgl. Bild 10) innerhalb vorgegebener Stautoleranzgrenzen (Stauziel),

- die Vergleichmäßigung von Abflußschwankungen, d.h. die geglättete Weitergabe von durch Störungen oder Stelleingriffe verursachten Änderungen des Abflusses an nachfolgende Stauhaltungen durch entsprechende Nutzung der Stauräume und

- die Möglichkeit, die Wehre und Kraftwerke gemäß einer vorgegebenen Betriebsvorschrift vollautomatisch zu betreiben.

Diesen Anforderungen an die Automatisierung stehen das komplexe, stark zeitvariante und räumlich verteilte Gebilde Stauraum sowie ein je nach Wasserangebot unterschiedliches Staustufenverhalten gegenüber. Durch Störeinflüsse, wie natürliche Abflußänderungen in Folge von Niederschlägen, Schneeschmelze oder Verdunstung, sowie künstliche Eingriffe durch den Schleusenbetrieb der Schiffahrt, Störungen im Betrieb des Wasserkraftwerks und durch die anliegende Industrie wird die Aufgabe zudem nachhaltig erschwert. Schließlich ist die Möglichkeit von Stelleingriffen in Form der Änderung des Abflusses über Turbinen und Wehre aus Aspekten der Sicherheit und Ökonomie stark eingeschränkt. Mitunter existierende Vorschriften, z. B., daß die Wehre einer speziellen Staustufe stündlich nur in wenigen Schritten verstellt werden dür-

Bild 10: Längsschnitt einer Stauhaltung.

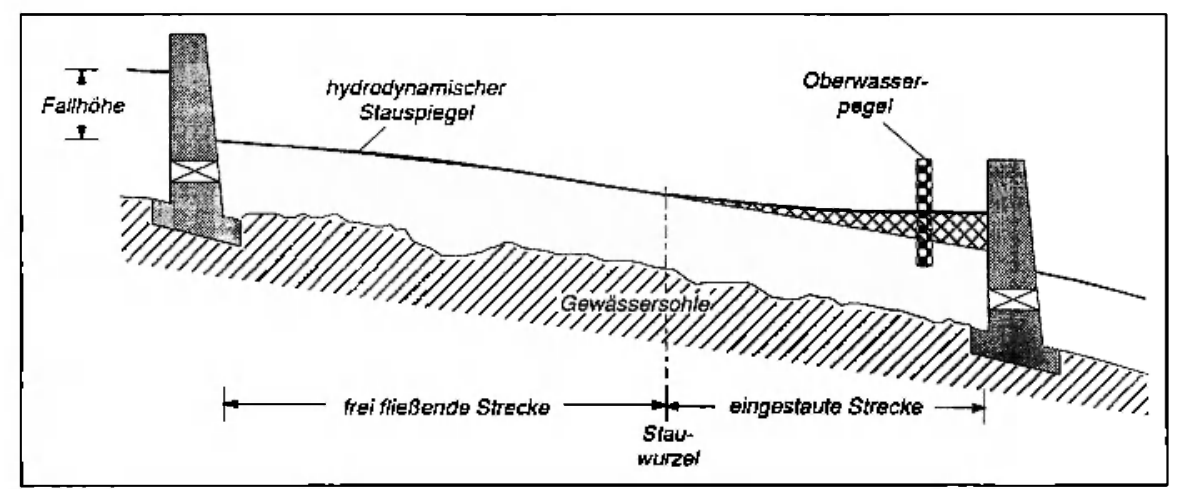


fen, stellen aus der Sicht der Regelungstechnik eine starke Einschränkung dar.

Aus der räumlichen und funktionellen Verteilung des zu automatisierenden Prozesses ergibt sich praktisch zwangsläufig eine hierarchisch dezentrale Struktur für die Automatisierungslösung. Ein Fluß, der in eine Reihe aufeinanderfolgender Staustufen eingeteilt ist, stellt zunächst eine Kaskade von Teilsystemen dar, die aus Gründen der Ausfallsicherheit und zur Gewährleistung kurzer Reaktionszeiten jedes für sich durch lokale Automatisierungseinheiten geregelt werden sollten. Aus der Gesamtheit der vielfach ineinandergreifenden und miteinander verkoppelten unterschiedlichen Automatisierungsfunktionen soll hier aus Gründen der Übersichtlichkeit lediglich die über einen Regelkreis mit Störgrößenaufschaltung realisierte Einhaltung des vorgegebenen Stauziels herausgegriffen werden (Bild 11).

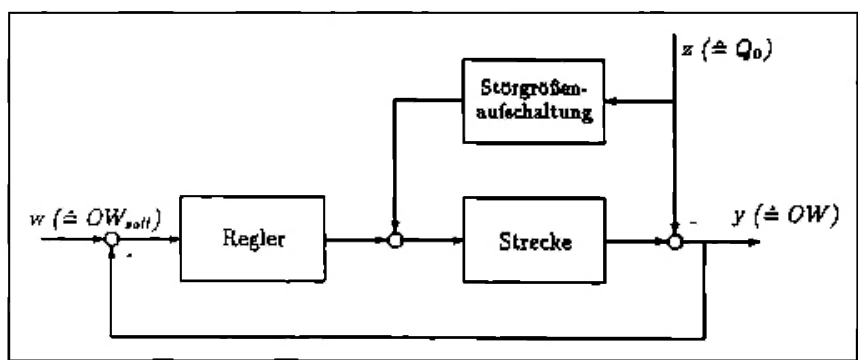

Bild 11: Regelkreis mit Störgrößenaufschaltung.

Der gesamte Regelkreis für den Oberwasserpegel einer Staustufe ist im Bild 12 als Blockschaltbild dargestellt. Zusätzlich zu der oben angeführten Struktur mit Störgrößenaufschaltung erkennt man einen unterlagerten Abflußregelkreis. Da der Istwert der Abflußregelung nicht direkt gemessen werden kann, muß er anhand der Fallhöhe und der Stellung der Wehre geschätzt werden. Die Filterung der Pegelmeßwerte dient dazu, neben den üblichen Meßungenauigkeiten auch Störungen, die beispielsweise durch die Schiffahrt hervorgerufen werden, zu glätten. Beim Entwurf der Automatisierungsanlagen ist zusätzlich zu berücksichtigen, $\mathrm{da} ß$ durch eventuelle Kommunikationsstörungen $z$. B. der Informationsfluß vom Oberlieger zeitweise ausfallen kann.

\subsection{Modellierungsaufgaben}

In dem im letzten Abschnitt beschriebenen Regelkreis für den Oberwasserstand einer Stauhaltung (Bild 12) wird an zwei Stellen ein mathematisches Modell der physikalischen Vorgänge benötigt. Zum einen ist dies bei der Schätzung des Wehr- und Turbinenabflusses der Fall und zum anderen bei der Realisierung der Störgrößenaufschaltung. Der nichtlineare Zusammenhang zwischen Leit- und Laufradstellung, Fallhöhe und Durchfluß kann den im Entwurfsprozeß erstellten bzw. in Abnahmeversuchen ermittelten Diagrammen (z. B. Muscheldiagramm bei Kaplanturbine) entnommen werden [12]. Je nachdem, ob ein Wehrverschluß unter-, durch- oder überströmt ist, vollkommener oder unvollkommener Überfall vorliegt, ergeben

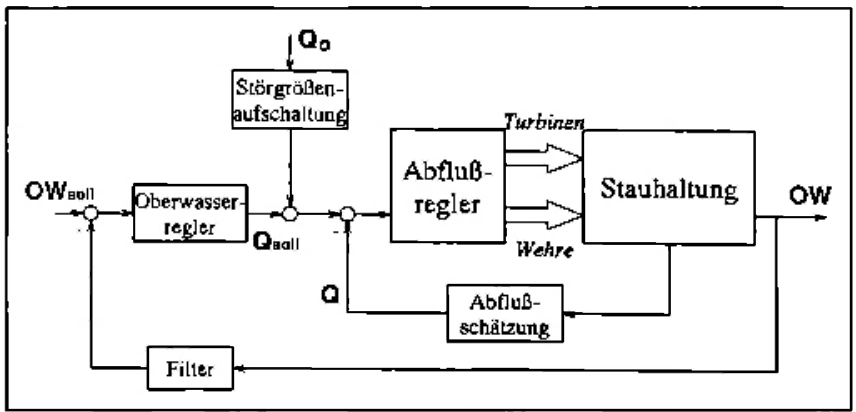

Bild 12: Blockschaltbild der Regelung des Oberwasserpegels.

sich unterschiedliche nichtlineare Zusammenhänge zwischen Wehrüberfall bzw. Wehröffnung sowie eventuell Fallhöhe einerseits und dem Durchfluß andererseits [13]. Diese Zusammenhänge werden näherungsweise über aus der Literatur bekannte Formeln mit geometrischen sowie abflußabhängigen Koeffizienten ermittelt oder im Rahmen von Modellversuchen am physikalischen Modell bestimmt. Die Dynamik der Abflußverstellung ist aufgrund der Antriebs- und Stellgrößenauslegung bekannt [12]. Während sich das Modell der Abflußorgane unter Berücksichtigung der aus Abnahme- und Modellversuchen gewonnene Daten relativ gut bestimmen läßt, bereitet die Modellbildung des verteilten Systems Stauraum für die Reglerauslegung sowie Störgrößenaufschaltung große Schwierigkeiten.

In einer exakten physikalischen Beschreibung der Stauhaltung müssen neben den dynamischen Eigenschaften des Fluids Wasser auch die morphologischen und geometrischen Eigenschaften natürlicher Flüsse sowie variierende Reibungseinflüsse berücksichtigt werden. Auch bei Beschränkung auf die Betrachtung einer eindimensionalen Längsströmung statt der dreidimensionalen Strömung, die in der Realität auftritt, erhält man zur Beschreibung der Kontinuitäts- und Impulsgleichungen bereits ein System nichtlinearer partieller St. Venant'scher Differentialgleichungen. Auf: der Basis eines so gewonnenen Modells wurde an der Bundesanstalt für Wasserbau erfolgreich das Verhalten einer realen Stauhaltung simuliert. Die Modellverifikation anhand von Naturversuchen zeigt durch Vergleich zwischen Messung und Simulationsrechnung eine sehr gute Übereinstimmung zwischen Modell und realem Prozeß sowohl hinsichtlich der Wasserspiegelhöhe im stationären als auch der Amplituden und Laufzeiten der Wellen im instationären Abflußfall [14]. Der numerische Aufwand bei diesen Simulationen ist aber sehr hoch und es werden zudem umfangreiche Daten über die Flußquerschnitte entlang des zu simulierenden Abschnitts benötigt.

Für den Einsatz im oben beschriebenen Regelkreis ist ein solches Modell schlecht geeignet; es ist numerisch zu aufwendig, und der Aufwand, der nötig ist, um das Modell an einen konkreten Prozeß mit seinen jeweiligen geometrischen Eigenschaften anzupassen, ist sehr groß. Wünschenswert aus der Sicht der Regelungstechnik ist statt dessen ein einfaches Modell mit konzentrierten Parametern, das eine hinreichend ge- 
naue Beschreibung des Prozesses bei gleichzeitiger Möglichkeit zur Anpassung an variierende Streckeneigenschaften bietet und dessen Struktur Rückschlüsse auf die Reglerauslegung gestattet.

Die Schwierigkeiten bei der Modellierung des dynamischen Verhaltens einer Flußstauhaltung ergeben sich unter anderem aus der Überlegung zweier grundsätzlich unterschiedlicher, den Wasserstand beeinflussender Effekte, die bei der Veränderung der Abflußverhältnisse auftreten. Nach einer beispielhaft angenommenen, sprungförmigen positiven Änderung des Zuflusses in die Stauhaltung (entspricht dem Oberliegerabfluß) erhöht sich in einer ersten Phase der Wasserspiegel entlang der freifließenden Strecke. Dabei stellt sich ein für den höheren Durchfluß zur Überwindung des größeren Reibungswiderstandes erforderliches höheres Gefälle ein. Das Volumen, das sich innerhalb der Retentionszeit $T_{R}$ aufbaut, wird als Retentionsvolumen $V_{R}$ bezeichnet. In einer darauf folgenden zweiten Phase steigt in Folge des Ungleichgewichts zwischen $\mathrm{Zu}$ - und $\mathrm{Ab}$ fluß der Stauhaltung der Oberwasserstand im Stauraum an. Neben diesen Speichereffekten beeinflussen auch die durch Zuflußänderungen verursachten Wellen, die als Schwall bzw. als Sunk bezeichnet werden und die sich gedämpft entlang der Stauhaltung ausbreiten, sowie deren Reflektion, den Wasserstand maßgeblich.

Bild 13 zeigt das Blockschaltbild eines aufgrund physikalischer Überlegungen des Autors B. Cuno gewonnenen, für die Regelung verwendeten linearisierten Modells [15]. Man erkennt sowohl im $\mathrm{Zu}$ - als auch im Abflußzweig die Parallelschaltung eines I-Glieds zur Modellierung der Speichereffekte und eines schwingfähigen linearen Gliedes als Modell der Welleneffekte. Für das schwingfähige Element wird dabei in der Regel ein $\mathrm{PT}_{2}$ - oder ein $\mathrm{PDT}_{2}$-Verhalten angenommen. Der Oberwasserstand ändert sich demnach aufgrund des Speicherverhaltens der Stauhaltung nach Ablauf der Retentionszeit $T_{R}$ und aufgrund der Welleneffekte, die sich um die Wellenlaufzeit $T_{L}$ verzögert auswirken. Das Prozeßmodell beschränkt sich somit auf die Darstellung von Zufluß, Abfluß und Wasserstand an den Endpunkten des Stauraums. Die zum Teil bedeutenden nichtlinearen Effekte im Stauraumverhalten werden durch die Ermittlung der vom jeweiligen Arbeitspunkt (Grundabfluß) abhängigen Parameter des linearisierten Modells berücksichtigt. Im Rahmen der Auslegung sowie des On-line-Betriebs der Automatisierungslö-

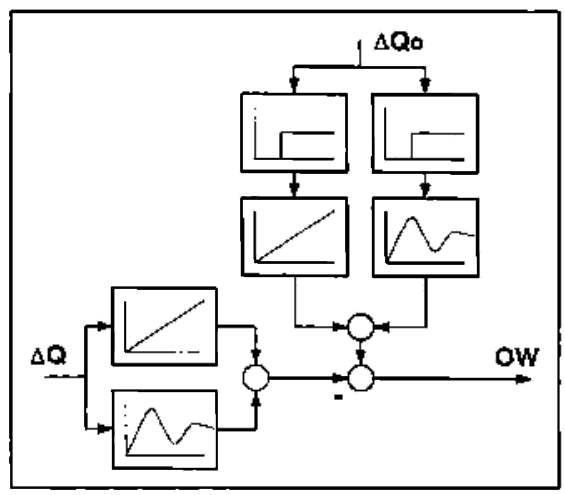

Bild 13: Modell einer Stauhaltung. sung werden die aktuell gültigen Arbeitspunkte ermittelt und zu einer gesteuerten Adaption der Regelparameter herangezogen. Da einerseits die Gradienten sowie die Amplituden der vom Automatisierungssystem bewirkten Abflußänderungen durch Vorgaben der Betreiber bzw. Genehmigungsbehörden auf relativ kleine Werte begrenzt sind (Stelleinwirkung) und andererseits naturbedingte Zuflußänderungen (bis auf die nur noch an wenigen Flüssen mögliche seltene Ausnahme des Eisstoßes) mit relativ großen Zeitkonstanten ablaufen bzw. bei Turbinenausfällen nur einen Teil des aktuellen Abflusses betreffen, ist die oben beschriebene Näherung zulässig und in zahlreichen Anwendungen erfolgreich eingesetzt. Auf die Berücksichtigung der bezüglich des Abflußverhaltens auftretenden Hystereseerscheinung im Stauraumverhalten (bei gleichem Wasserstand ist das Wasserspiegelgefälle bei steigendem Wasserstand größer als bei fallendem) kann im Rahmen der Regelkreisauslegung aufgrund bisheriger Erfahrungen verzichtet werden.

\section{Untersuchungen}

Im Rahmen der Diplomarbeit des Autors P. Marenbach wurde untersucht, inwieweit sich mittels des hier vorgestellten Ansatzes zur selbstorganisierenden Modellbildung ein vernünftiges Modell des dynamischen Verhaltens des Oberwasserstands einer Flußstauhaltung überhaupt generieren läßt und inwieweit sich dabei Ähnlichkeiten oder Unterschiede zu der im Abschnitt 4.2 dargestellten Modellierung ergeben.

Wie auch bei anderen selbstlernenden Verfahren -z. B. Neuronalen Netzen - ist eine zentrale Bedingung für eine erfolgreiche Modellbildung, daß variantenreiche Datensätze des zu modellièrenden Prozesseses vor̈liegen. Entsprechende Datensätze für die Modellbildung einer Flußstauhaltung zu gewinnen, ist aus praktischen Gründen nur in seltenen Fällen möglich, da die Betreiber der Anlagen nicht in der Lage oder nicht willens sind, einen Flußabschnitt für regelungstechnische Versuchsreihen im Hinblick auf eine Gefährdung von Schiffahrt, Energieerzeugung und Betriebssicherheit des Flusses sowie Kraftwerks zur Verfügung zu stellen. Jedoch steht bei der Bundesanstalt für Wasserbau in Karlsruhe für derartige Versuche zum einen ein physikalisches Modell, bestehend aus zwei im Modellmaßstab nachgebildeten Staustufen, zur Verfügung und zum anderen ein Programmpaket, mit dem Flußstauhaltungen auf Basis der Lösung der verteilten St. Venant'schen Differentialgleichungen numerisch simuliert werden können. Als Beispielprozeß wurde die Stauhaltung Bad Säckingen mit dem Rheinkraftwerk Säckingen gewählt.

Bei der Untersuchung, die im folgenden betrachtet werden soll, wurden 500 Individuen über 50 Generationen betrachtet, im „Modellbaukasten“ waren im Hinblick auf die Anmerkungen zur Linearisierbarkeit im vorigen Abschnitt nur lineare Blöcke enthalten und die Parameteroptimierung erfolgte mit dem Verfahren nach Hooke und Jeeves [10]. Als Prozeßdaten wurden 

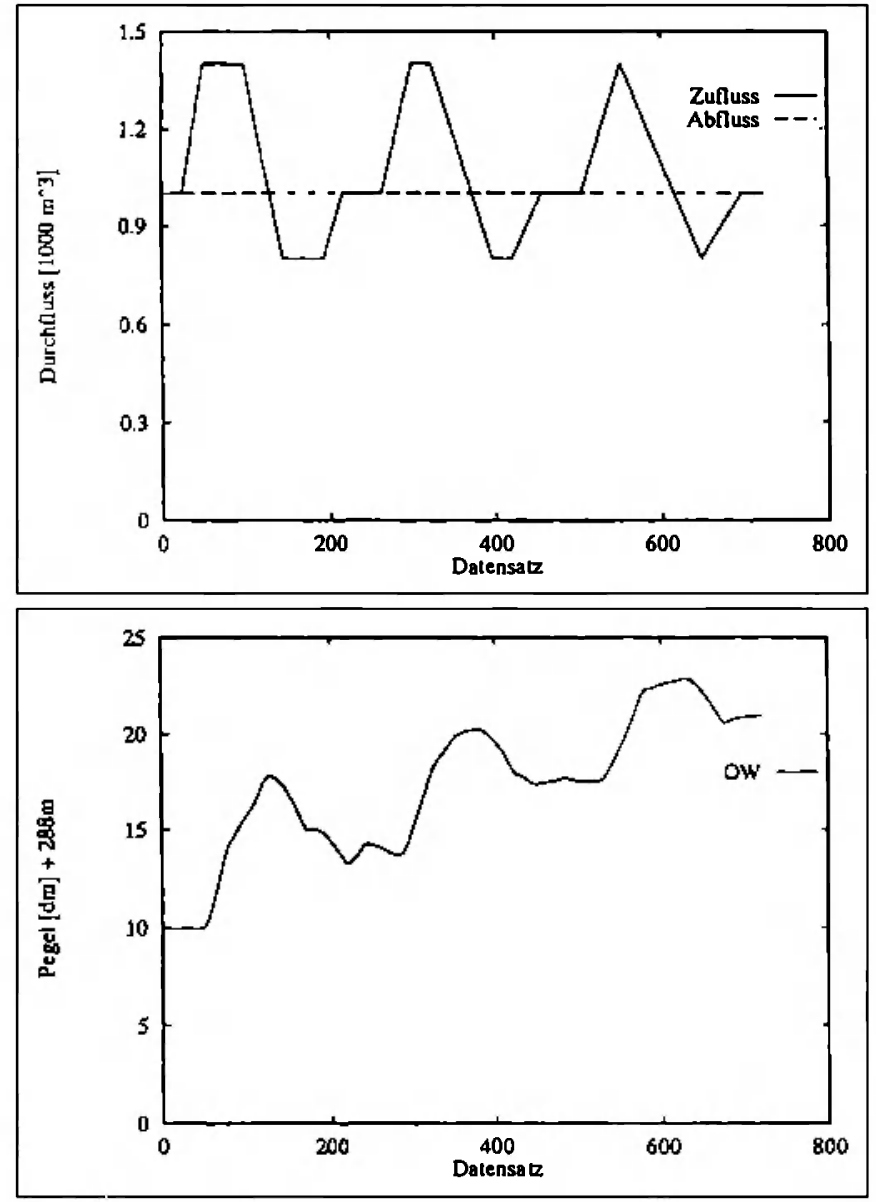

Bild 14: Trainingssignal I mit wechselndem Zufluß: Anregungssignalc (oben) und Ausgangsgröße (unten).

zwei Trainingssignale verwendet; dabei sind jeweils $\mathrm{Zu}-$ und $A b f l u ß$ der Stauhaltung die Eingangsgrößen und Ausgangsgröße ist der Oberwasserpegel an der Staustufe. An dem im Bild 14 wiedergegebenen Verlauf, bei dem insgesamt mehr Wasser in die Stauhaltung hinein als heraus fließt, läßt sich sehr gut der Speichereffekt beobachten, und die je einmalige kurzzeitige Änderung von Zufluß und Abfluß, die der Verlauf im Bild 15 zeigt, eignet sich sehr gut zum Erkennen der ebenfalls beschriebenen Welleneffekte.

Das jeweils beste Individuum einer betrachteten Generation stellt das bis zu dieser Generation erzielte Ergebnis der selbstorganisierenden Modellbildung dar. In einem Grundlagenartikel, wie dem vorliegenden, ist es leider nicht möglich, alle während des Evolutionsprozesses zusammenwirkenden Effekte ausführlich zu beschreiben. Betrachtet man aber die Entwicklung dieses besten Individuums im Laufe der Generationen, so läßt sich ein erster Eindruck von der Arbeitsweise des hier vorgestellten Verfahrens gewinnen.

Nach 10 Generationen ergab sich das noch sehr einfache im Bild 16 dargestellte Modell. Unter dem Blockschaltbild sind der sich mit diesem Modell in der Simulation ergebende Verlauf des Oberwasserpegels und zum Vergleich der entsprechende Sollverlauf aus Bild 14 aufgetragen. Dieses Modell gibt lediglich das Speicherverhalten der Stauhaltung wieder und würde sicherlich auch bei einer induktiven Modellbildung den
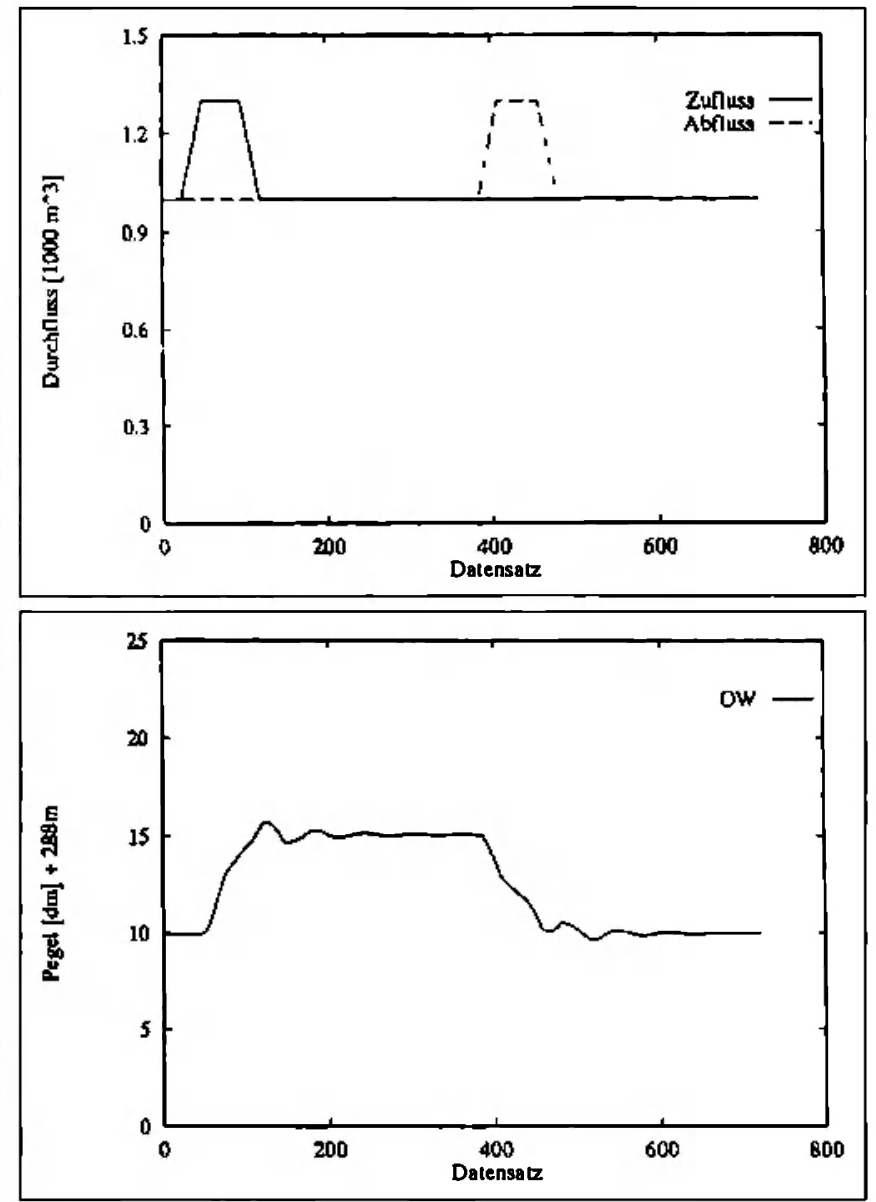

Bild 15: Trainingssignal II mit je einmaliger Änderung von Zufluß und Abfluß: Anregungssignale (oben) und Ausgangssignale (unten).

Ausgangspunkt darstellen. Das Modell, das das beste Individuum der 25. Generation repräsentierte, sowie den Vergleich zwischen dem angestrebten Sollverlauf

Bild 16: Bestes Individuum nach 10 Generationen: Blockschaltbild (oben) sowie Soll- und Istverlauf (unten).
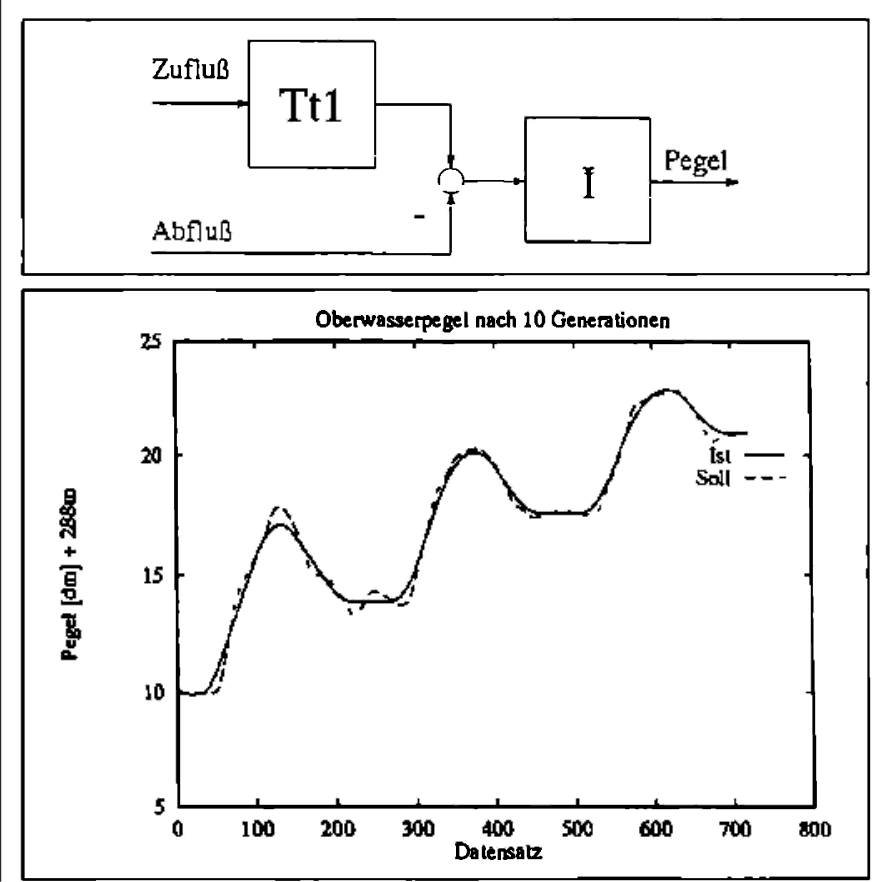

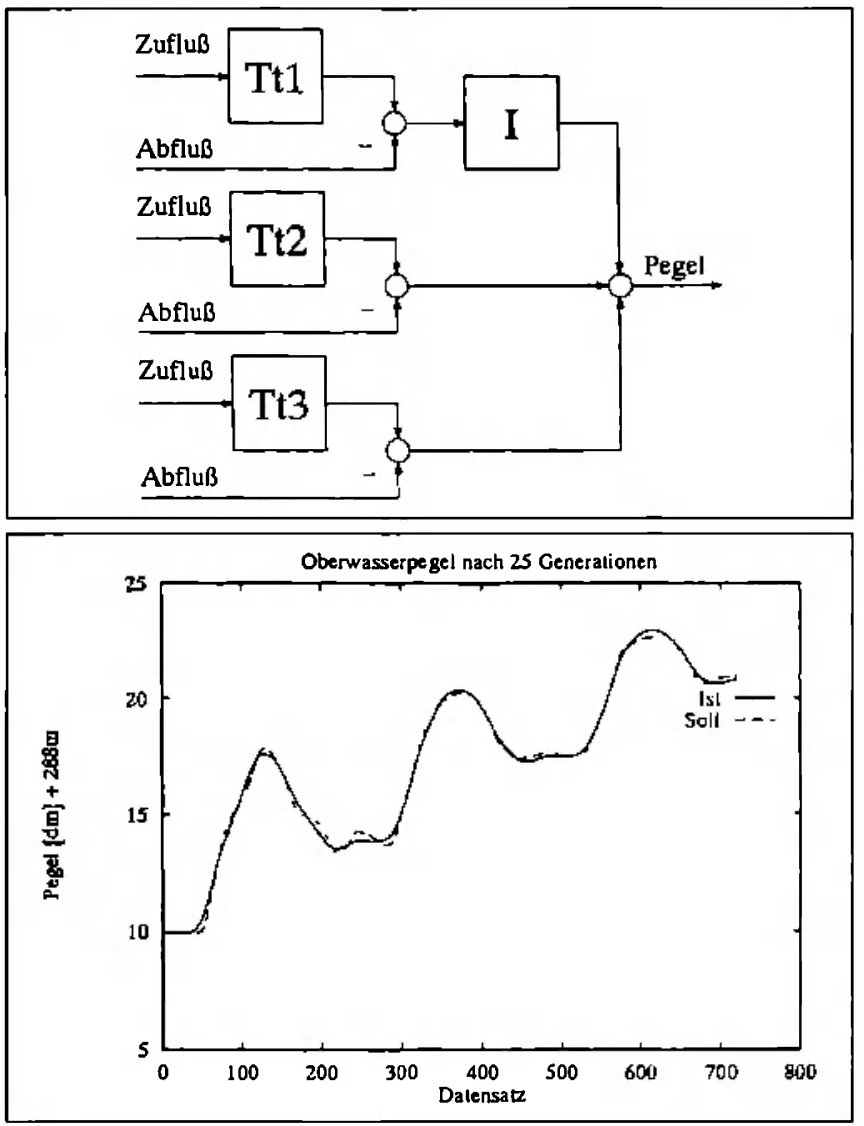

Bild 17: Bestes Individuum nach 25 Generationen: Blockschaltbild (oben) sowie Soll- und Istverlauf (unten).

und dem Verlauf, der sich mit diesem Modell ergibt, zeigt Bild 17. Man erkennt dort im Vergleich zu dem Modell aus Bild 16 zwei zusätzliche $Z$ weige, in denen die Differenz aus Zu- und Abfluß mit unterschiedlichen Totzeiten zu dem Ergebnis der Integration addiert wird. Diese Struktur nähert das Sollverhalten schon deutlich besser an, es läßt sich allerdings anhand physikalischer Überlegungen nicht motivieren und ist noch nicht in der Lage, die im Sollverlauf auftretenden Schwingungen wiederzugeben. In der 50 . Generation lag schließlich die im Bild 18 wiedergegebene Struktur vor. Dieses Modell zeigt, wie man an dem unter dem Blockschaltbild dargestellten Verlauf erkennt, eine sehr gute Übereinstimmung mit den beim Training verwendeten Sollverläufen. Die von Cuno vorgeschlagene und im Bild 13 dargestellte Struktur enthält bei gleichen Eingangs-/Ausgangsgrößen - die Kleinsignalgröße $Q_{0}$ entspricht der Bezeichnung Zufluß, $Q$ dem $A$ bfluß und $O W$ dem Pegel (siehe dazu auch die ausführlichen Erläuterungen im Abschnitt 4.2) - ein zusätzliches $\mathrm{PDT}_{2}$ Glied im Abflußzweig, das sich vom PDT $_{2}$-Glied im Zuflußzweig unterscheidet. Dadurch ergibt sich bei optimierten Parametern gegenüber der gefundenen Struktur noch eine gewisse Verbesserung in der Anpassung an den realen Verlauf, die aber unterhalb der Auflösungsgenauigkeit der graphischen Darstellung im

Bild 19: Versuch mit alternativem Datensatz: Anregungssignale (oben) und Vergleich von Soll- und Istverlauf der Ausgangsgröße (unten).
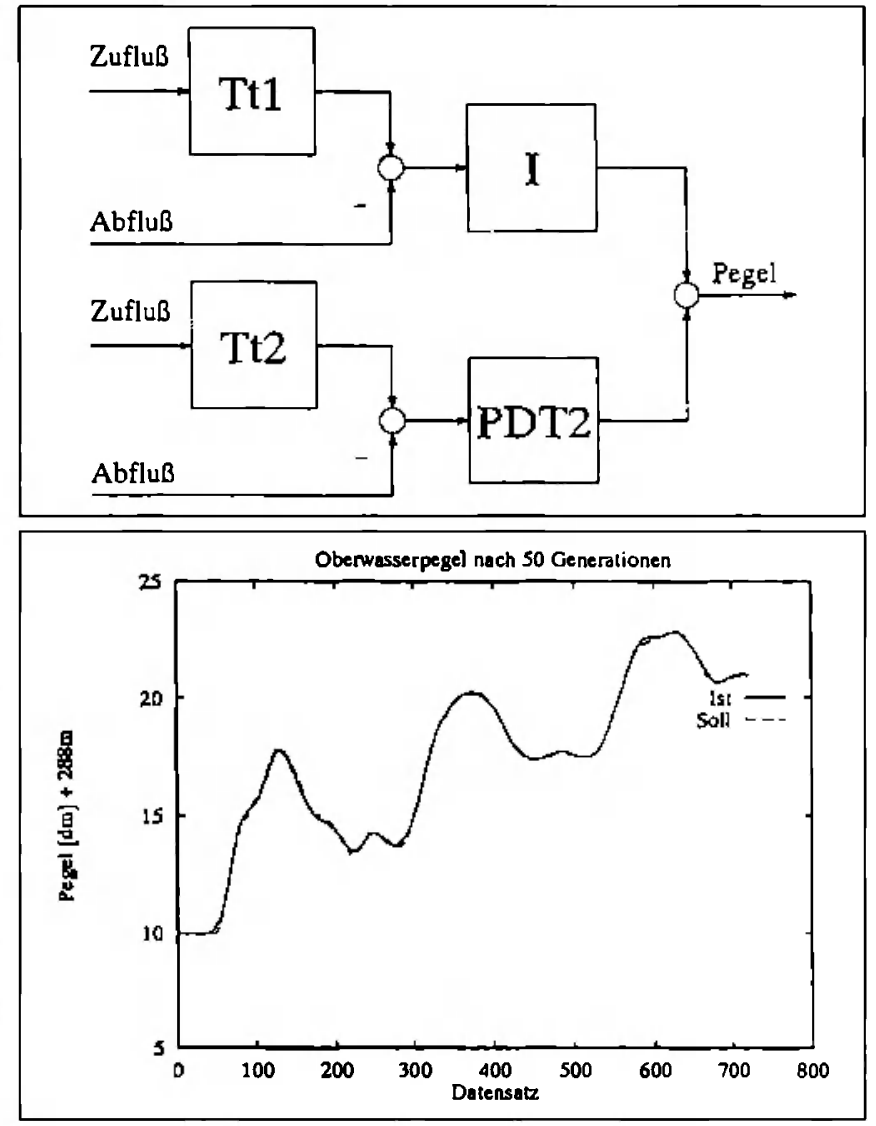

Bild 18: Bestes Individuum nach 50 Generationen: Blockschaltbild (oben) sowie Soll- und Istverlauf (unten).
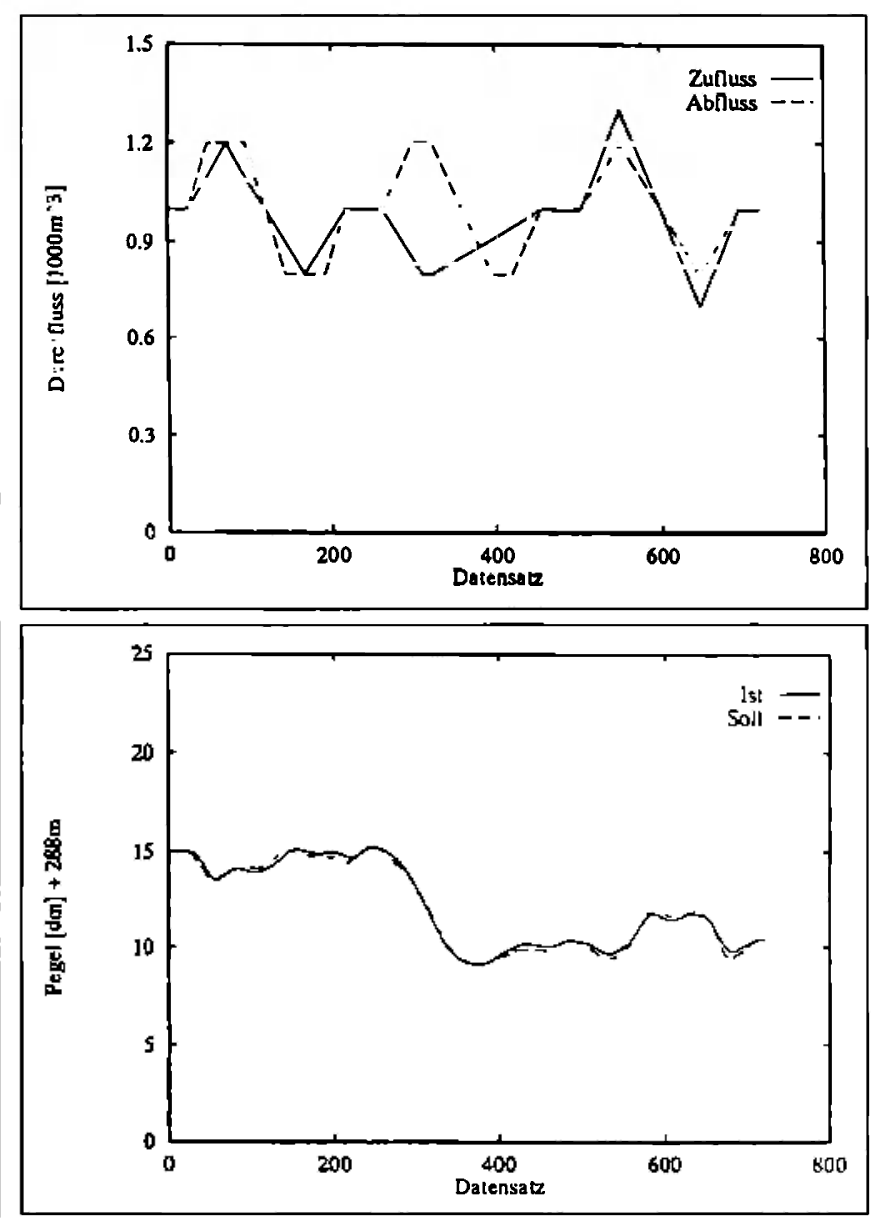
Bild 18 (unten) liegt. Bei der selbstorganisierenden Modellierung wird diese prinzipiell mögliche Ergänzung während des Evolutionsprozesses vermutlich infolge der heuristischen Gleichung (1) zur Bestimmung der Fitneß verworfen, da die im Komplexitätsmaß $K_{i}$ enthaltene ,Strafe" für ein zusätzliches $\mathrm{PDT}_{2}$-Glied höher ausfällt als die Verringerung des Fehlermaßes $E_{i}$ und sich demzufolge die Fitneß verschlechtert.

Bekanntermaßen zeigt sich die wahre Qualität eines erlernten Modells erst, wenn man dieses Modell an einem Datensatz erprobt, an dem nicht explizit gelernt wurde. Im Bild 19 erkennt man, daß man mit dem Modell, das nach 50 Generationen vorlag, auch bei einem anderen Datensatz respektable Ergebnisse erhält. Hierbei wurde lediglich der Anfangswert des Integrators geeignet verändert, um den Fehler durch die unterschiedlichen Anfangswerte auszugleichen.

\section{Zusammenfassung und Ausblick}

Die Erstellung eines geeigneten dynamischen Prozeßmodells ist eine der wesentlichen Aufgabenstellungen der Regelungstechnik. Das Modell stellt stets den Ausgangspunkt für Entwurf und Auslegung von Regelstrategien dar und ist daher von zentraler Bedeutung. Die analytische Modellbildung ist in der Regel ein mühsamer, langwieriger Prozeß. Aus diesem Grund werden seit langem selbstlernende datengetriebene Verfahren zur automatischen Generierung von Modellen untersucht. Derartige Verfahren weichen in der Art, in der sie das Prozeßverhalten beschreiben, von der sonst üblichen, physikalisch fundierten Bechreibungsform durch Differentialgleichungen $a b$. Bewährte Verfahren zur Synthese und Auslegung von Regelsystemen lassen sich auf derartige Modelle nicht anwenden.

In diesem Beitrag wurde ein neuartiger Ansatz vorgestellt, mit dem es möglich ist, selbstorganisierend dynamische Prozeßmodelle in genau der strukturierten Form zu generieren, die in der Regelungstechnik üblich ist und die sich für die systemtheoretische Analyse des Prozesses und die darauf aufbauende Reglersynthese in der Vergangenheit bewährt hat. Dieser Ansatz kombiniert die evolutionäre Vorgehensweise des ,genetischen Programmierens" zur Suche geeigneter Modellstrukturen mit konventionellen Suchverfähren zur lokalen Parameteroptimierung.

Bei Untersuchungen zur Modellbildung des dynamischen Verhaltens einer Fluß-Stauhaltung wurden erste erfolgversprechende Ergebnisse bei der Synthese eines Modells aus linearen Bausteinen erzielt, die bei vergleichenden Experimenten mit konventionellen Identifikationsverfahren nicht erreicht werden konnten.

Neben der rechenzeitoptimalen Implementierung des beschriebenen Ansatzes in Form eines leicht bedienbaren und vielseitig anwendbaren ,Werkzeugkastens" wird sich eine der nächsten Arbeiten mit der Integration effizienter Parameteridentifikationsverfahren anstelle des bislang verwendeten Suchverfahrens nach Hooke und Jeeves beschäftigen, da hiervon sowohl deutliche Verbesserungen des Laufzeitverhaltens als auch der Qualität der erzeugten Modelle erwartet werden.

Aktuelle praktische Untersuchungen beschäftigen sich mit der Synthese des Modells einer biotechnologischen Enzymfermentation, bei dem, bedingt durch die auftretenden Repressionsmechanismen, eine Reihe von nichtlinearen Bausteinen, z. B. zur Modellierung der Substratinhibierung, integriert werden müssen; diese Versuche sind jedoch noch nicht soweit fortgeschritten, daß sie an dieser Stelle bereits ausführlich dargestellt werden könnten.

\section{Danksagung}

Die Autoren bedanken sich herzlich bei Herrn Dipl.-Ing. Karlheinz Metzger, Betriebsdirektor des Rheinkraftwerkes Säckingen, sowie bei Herrn Dipl.-Ing. Stephan Theobald, Abteilung Wasserbau/Hydraulik der Bundesanstalt für Wasserbau (BAW), Karlsruhe, für die fruchtvolle Zusammenarbeit und besonders für die Bereitstellung der Ergebnisse der dynamischen Modellversuche sowie der Simulationsberechnungen.

\section{Literatur}

[1] Föllinger, $O$.: Regelungstechnik. 7. Auflage. Hüthig Buch Verlag, Heidelberg 1992.

[2] Tolle, $H$., und Ersü, E.: Neurocontrol. Nummer 172 in Lecture Notes in Control and Information Sciences. Springer-Verlag, Berlin, Heidelberg, New York 1992.

[3] Isermann, $R$.: Identifikation dynamischer Systeme. Band $1 \& 2$. 2. Auflage. Springer-Verlag, Berlin 1992.

[4] van Hentenryck, $P$.: Constraint satisfaction in logic programming. MIT Press, Cambridge, Massachusetts 1989.

[5] Schittkowski, K.: Nonlinear Programming Codes - Information, Tests, Performance. Springer-Verlag, Berlin, Heidelberg, New York 1980

[6] Rechenberg, I.: Evolutionsstrategiè - Optimièrung technischer Systeme nach Prinzipien der biologischen Evolution. Frommann-Verlag 1973.

[7] Holland, J.H.: Adaptation in natural an artificial systems. The University of Michigan Press 1975.

[8] Sprave, J.: Zelluläre Evolutionäre Algorithmen zur Parameteroptimierung. In: Hofestädt, R., Krückeberg, F. und Lengauer, $T$. (Hrsg.): Informatik in den Biowissenschaften, Informatik aktuell. S. 111-120, Bonn, Februar 1993, Springer-Verlag.

[9] Koza, J.R.: Genetic Programming: On the Programming of Computers by Means of Natural Selection. The MIT Press, Cambridge, Massachusetts 1992.

[10] Hooke, R., und Jeeves, T.A.: Direct Search: Solution of Numerical and Statistical Problems. Journal of the Association of Computing Machinery (1961) S. 212-224.

[11] Goldberg, D.E., und $D e b, K$.: A comparative analysis of selection schemes. In: Rawlins, G.J.R. (Hrsg.): Foundations of Genetic Algorithms. Morgan Kaufmann Publishing 1991.

[12] Raabe, J.: Hydro Power. The Design, Use and Function of Hydromechanical, Hydraulic and Electrical Equipment. VDIVerlag, Düsseldor 1985

[13] Naudascher, E.: Hydraulik der Gerinne und Gerinnebauwerke. Springer-Verlag, Wien, New York 1987.

[14] Nestmann, F., und Theobald, S.: Numerisches Modell zur Steuerung und Regelung einer Staustufenkette am Beispiel von Rhein und Neckar. Wasserwirtschaft (1994) S. 72-78.

[15] Cuno, B.: Entwurf des Automatisierungssystems für eine Stauhaltungskette. Technischer Bericht, AEG Frankfurt 1985.

\section{Manuskripteingang: 2. Mai 1994.}

